\title{
FROM THE NON-ABELIAN TO THE SCALAR TWO-DIMENSIONAL TODA LATTICE
}

\author{
CORNELIA SCHIEBOLD \\ Mathematisches Institut, Friedrich-Schiller-Universität Jena, 07737 Jena, Germany \\ e-mail: cornelia@minet.uni-jena.de
}

(Received 30 October, 2003; accepted 13 September, 2004)

\begin{abstract}
We extend a solution method used for the one-dimensional Toda lattice in [1], [2] to the two-dimensional Toda lattice. The idea is

1. to study the lattice not with values in $\mathbb{C}$ but in the Banach algebra $\mathcal{L}$ of bounded operators and

2. to derive solutions of the original lattice ( $\mathbb{C}$-solutions) by applying a functional $\tau$ to the $\mathcal{L}$-solutions constructed in 1 .

The main advantage of this process is that the derived solution still contains an element of $\mathcal{L}$ as parameter that may be chosen arbitrarily. Therefore, plugging in different types of operators, we can systematically construct a huge variety of solutions.

In the second part we focus on applications. We start by rederiving line-solitons and briefly discuss discrete resonance phenomena. Moreover, we are able to find conditions under which it is possible to superpose even countably many line-solitons.
\end{abstract}

2000 Mathematics Subject Classification. 35Q58, 32A65.

1. Introduction. In this article we study the so-called two-dimensional Toda lattice

$$
\frac{\partial^{2}}{\partial x \partial y} w_{n}=\exp \left(w_{n}-w_{n-1}\right)-\exp \left(w_{n+1}-w_{n}\right)
$$

where $w_{n}=w_{n}(x, y), n \in \mathbb{Z},(x, y) \in \mathbb{R}^{2}$, which actually has been already considered by Darboux [3] in 1915. For further information and related results we refer to [4], [5], [6], [7], [8], [9] and [10].

The present work is based on an operator theoretic approach developed by Aden, Carl, and the author [11], [1], [12], that was inspired by pioneering work of Marchenko [13].

First we consider a non-abelian two-dimensional Toda lattice taking its values in the Banach algebra of bounded operators. In Theorem 2.1 we provide an explicit operator-valued solution, which is formally similar to the line-soliton but contains two operator-valued commuting parameters. From this we derive in Theorem 2.3 the solution formula for the scalar two-dimensional Toda lattice which will be fundamental for our applications. The decisive gain is that this solution formula still depends on the same operator-valued parameters as before. (See [1] for a comparable treatment of the one-dimensional Toda lattice.)

The above procedure is given in the framework of Banach spaces. But many of the salient features can already be seen in the finite-dimensional case. For the sake of 
illustration we rederive $N$-solitons (see also [14], [15]). As a more interesting finitedimensional application, we try to give the reader a first impression of how to use our method in the study of resonantly interacting solitons; i.e., solutions similar to the Miles structures of the Kadomtsev-Petviashvili equation (see [16], [17], [18], [19]). Here a comparison with our treatment of the one-dimensional Toda-lattice is instructive. In this case there is only one operator-valued parameter in the solution formula, and, as shown in [1], [2], the finite-dimensional case reduces completely to matrices in Jordan form yielding the so-called negatons. The case of the two-dimensional Toda lattice seems to be essentially more intricate as can already be seen for the simplest Miles structures. (See Section 3.2.)

Our main application concerns the problem of countable superpositions of solitons as raised and pursued by Gesztesy and collaborators [20], [21]. Here infinitedimensional methods become truely crucial. The approach of [20], [21] consists in a direct passage to the limit requiring a good deal of hard analysis. In our approach, the difficulty reduces to summation properties of operators on Banach spaces, a topic which was systematically developed in [22], [23]. For a comparison of the two methods in the $\mathrm{KdV}$ case we refer to [20], [24]. To the best of our knowledge Theorem 4.3 is the first result in this direction for two-dimensional discrete equations.

2. Operator-theoretic treatment. For convenience, we use the dependent variable transform $v_{n}=\exp \left(w_{n}\right)-1$ to rewrite the lattice in the form

$$
\frac{\partial^{2}}{\partial x \partial y} \log \left(1+v_{n}\right)=\frac{1+v_{n}}{1+v_{n-1}}-\frac{1+v_{n+1}}{1+v_{n}} .
$$

2.1. Non-abelian two-dimensional Toda lattice. Let us now consider the nonabelian version of the two-dimensional Toda lattice,

$$
\frac{\partial}{\partial y}\left(\left(1+V_{n}\right)^{-1} \frac{\partial}{\partial x} V_{n}\right)=\left(1+V_{n-1}\right)^{-1}\left(1+V_{n}\right)-\left(1+V_{n}\right)^{-1}\left(1+V_{n+1}\right),
$$

where $V_{n}=V_{n}(x, y)$ takes values in the space of bounded operators $\mathcal{L}(E)$ on some Banach space $E$. In view of the applications in Section 4 the statements will be given for possibly infinite-dimensional Banach spaces. To grasp the main argument, it is enough to understand the finite-dimensional case.

An explicit solution of (3) is provided by the following result.

THEOREM 2.1. Let $A, B$ be constant invertible operators on some Banach space $E$ with $[A, B]=0$. If the family of operators $L_{n}(x, y)$ solves the following set of linear equations

$$
L_{n+1}=(A B) L_{n}, \quad \frac{\partial}{\partial x} L_{n}=\left(A-B^{-1}\right) L_{n} \quad \text { and } \quad \frac{\partial}{\partial y} L_{n}=\left(A^{-1}-B\right) L_{n}
$$

and if $\left(1+L_{n}\right)$ is invertible for all $n \in \mathbb{Z}$ and all $(x, y) \in \Omega$, where $\Omega$ is an open subset of $\mathbb{R}^{2}$, then a solution of (3) on $\Omega$ is given by

$$
V_{n}:=\left(1+L_{n}\right)^{-1}\left(A L_{n} B-L_{n}\right)
$$

Non-abelian Toda lattices are known in the literature. For example in [7], the matrix-version of (3) was studied from the Darboux transformation point of view. 
Observe that if $L_{n+1}=(A B) L_{n}$ holds for all $n \in \mathbb{Z}$, then the differential equations in (4) need only to be checked for some $n_{0}$.

Proof. First note that

$$
\begin{aligned}
1+V_{n} & =\left(1+L_{n}\right)^{-1}\left(1+A L_{n} B\right)=\left(1+L_{n}\right)^{-1}\left(1+B^{-1} \cdot A B L_{n} \cdot B\right) \\
& =\left(1+L_{n}\right)^{-1} B^{-1}\left(1+L_{n+1}\right) B
\end{aligned}
$$

and thus

$$
\left(1+V_{n}\right)^{-1}=B^{-1}\left(1+L_{n+1}\right)^{-1} B\left(1+L_{n}\right)
$$

By the usual product rule and the non-abelian differentiation of the inverse operator of $T=T(x)$ with respect to $x,\left(T^{-1}\right)_{x}=-T^{-1} T_{x} T^{-1}$, we get

$$
\begin{aligned}
\frac{\partial}{\partial x} V_{n}= & -\left(1+L_{n}\right)^{-1}\left(A-B^{-1}\right) L_{n}\left(1+L_{n}\right)^{-1}\left(A L_{n} B-L_{n}\right) \\
& +\left(1+L_{n}\right)^{-1}\left(A-B^{-1}\right)\left(A L_{n} B-L_{n}\right) \\
= & \left(1+L_{n}\right)^{-1}\left(A-B^{-1}\right)\left(-\left(1+L_{n}\right)^{-1} L_{n}+1\right)\left(A L_{n} B-L_{n}\right) \\
= & \left(1+L_{n}\right)^{-1}\left(A-B^{-1}\right) V_{n}
\end{aligned}
$$

and, analogously,

$$
\frac{\partial}{\partial y} V_{n}=\left(1+L_{n}\right)^{-1}\left(A^{-1}-B\right) V_{n}
$$

From (6), (7) we have $\left(1+V_{n}\right)^{-1} \frac{\partial}{\partial x} V_{n}=B^{-1}\left(1+L_{n+1}\right)^{-1}(A B-1) V_{n}$, and thus the left-hand side of $(3)$ reads

$$
\begin{aligned}
\frac{\partial}{\partial y} & \left(\left(1+V_{n}\right)^{-1} \frac{\partial}{\partial x} V_{n}\right)=B^{-1}\left(1+L_{n+1}\right)^{-1}(A B-1)\left(\left(1+L_{n}\right)^{-1}\left(A^{-1}-B\right) V_{n}\right) \\
& +B^{-1}\left(-\left(1+L_{n+1}\right)^{-1}\left(A^{-1}-B\right) L_{n+1}\left(1+L_{n+1}\right)^{-1}\right)(A B-1) V_{n} \\
= & B^{-1}\left(1+L_{n+1}\right)^{-1}(A B-1)\left(A^{-1} L_{n+1}\left(1+L_{n+1}\right)^{-1}-\left(1+L_{n}\right)^{-1} A^{-1}\right)(A B-1) V_{n} .
\end{aligned}
$$

Calculating the expression in the bracket

$$
\begin{aligned}
A^{-1} & L_{n+1}\left(1+L_{n+1}\right)^{-1}-\left(1+L_{n}\right)^{-1} A^{-1} \\
& =\left(1+L_{n}\right)^{-1}\left(\left(1+L_{n}\right) A^{-1} L_{n+1}-A^{-1}\left(1+L_{n+1}\right)\right)\left(1+L_{n+1}\right)^{-1} \\
& =\left(1+L_{n}\right)^{-1}\left(L_{n} A^{-1} L_{n+1}-A^{-1}\right)\left(1+L_{n+1}\right)^{-1} \\
& =\left(1+L_{n}\right)^{-1}\left(L_{n} A^{-1}(A B) L_{n}-A^{-1}\right)\left(1+L_{n+1}\right)^{-1} \\
& =\left(1+L_{n}\right)^{-1}\left(L_{n} B L_{n}-A^{-1}\right)\left(1+L_{n+1}\right)^{-1},
\end{aligned}
$$

we finally obtain

$$
\begin{aligned}
& \frac{\partial}{\partial y}\left(\left(1+V_{n}\right)^{-1} \frac{\partial}{\partial x} V_{n}\right) \\
& \quad=B^{-1}\left(1+L_{n+1}\right)^{-1}(A B-1)\left(1+L_{n}\right)^{-1}\left(L_{n} B L_{n}-A^{-1}\right)\left(1+L_{n+1}\right)^{-1}(A B-1) V_{n} .
\end{aligned}
$$


Now we show that this expression coincides with the right-hand side of (3). Using again (6) as well as the identities

$$
\begin{aligned}
& V_{n}-V_{n-1}=\left(1-\left(1+L_{n-1}\right)^{-1}(A B)^{-1}\left(1+L_{n}\right)\right) V_{n}=\left(1+L_{n-1}\right)^{-1}\left(1-(A B)^{-1}\right) V_{n} \\
& V_{n+1}-V_{n}=\left(\left(1+L_{n+1}\right)^{-1}(A B)\left(1+L_{n}\right)-1\right) V_{n}=\left(1+L_{n+1}\right)^{-1}((A B)-1) V_{n}
\end{aligned}
$$

we get

$$
\begin{aligned}
(1+ & \left.V_{n-1}\right)^{-1}\left(1+V_{n}\right)-\left(1+V_{n}\right)^{-1}\left(1+V_{n+1}\right) \\
= & \left(1+V_{n-1}\right)^{-1}\left(V_{n}-V_{n-1}\right)-\left(1+V_{n}\right)^{-1}\left(V_{n+1}-V_{n}\right) \\
= & B^{-1}\left(1+L_{n}\right)^{-1} B\left(1+L_{n-1}\right) \cdot\left(1+L_{n-1}\right)^{-1}\left(1-(A B)^{-1}\right) V_{n} \\
& -B^{-1}\left(1+L_{n+1}\right)^{-1} B\left(1+L_{n}\right) \cdot\left(1+L_{n+1}\right)^{-1}((A B)-1) V_{n} \\
= & B^{-1}\left(\left(1+L_{n}\right)^{-1} B(A B)^{-1}-\left(1+L_{n+1}\right)^{-1} B\left(1+L_{n}\right)\left(1+L_{n+1}\right)^{-1}\right)(A B-1) V_{n} \\
= & B^{-1}\left(\left(1+L_{n}\right)^{-1} A^{-1}\left(1+L_{n+1}\right)-\left(1+L_{n+1}\right)^{-1} B\left(1+L_{n}\right)\right)\left(1+L_{n+1}\right)^{-1}(A B-1) V_{n} .
\end{aligned}
$$

As for the expression in the bracket, we use

$$
\begin{aligned}
\left(1+L_{n}\right)^{-1}-\left(1+L_{n+1}\right)^{-1} & =\left(1+L_{n+1}\right)^{-1}\left(\left(1+L_{n+1}\right)-\left(1+L_{n}\right)\right)\left(1+L_{n}\right)^{-1} \\
& =\left(1+L_{n+1}\right)^{-1}\left((A B-1) L_{n}\right)\left(1+L_{n}\right)^{-1}
\end{aligned}
$$

yielding $\left(1+L_{n}\right)^{-1}=\left(1+L_{n+1}\right)^{-1}\left(1+(A B-1)\left(1+L_{n}\right)^{-1} L_{n}\right)$.

Therefore

$$
\begin{aligned}
(1 & \left.+L_{n}\right)^{-1} A^{-1}\left(1+L_{n+1}\right)-\left(1+L_{n+1}\right)^{-1} B\left(1+L_{n}\right) \\
& =\left(1+L_{n+1}\right)^{-1}\left(\left(1+(A B-1)\left(1+L_{n}\right)^{-1} L_{n}\right) A^{-1}\left(1+L_{n+1}\right)-B\left(1+L_{n}\right)\right) \\
& =\left(1+L_{n+1}\right)^{-1}\left(\left(A^{-1}-B\right)+(A B-1)\left(1+L_{n}\right)^{-1} L_{n} A^{-1}\left(1+L_{n+1}\right)\right) \\
& =\left(1+L_{n+1}\right)^{-1}(A B-1)\left(-A^{-1}+\left(1+L_{n}\right)^{-1} L_{n} A^{-1}\left(1+L_{n+1}\right)\right) \\
& =\left(1+L_{n+1}\right)^{-1}(A B-1)\left(1+L_{n}\right)^{-1}\left(-\left(1+L_{n}\right) A^{-1}+L_{n} A^{-1}\left(1+L_{n+1}\right)\right) \\
& =\left(1+L_{n+1}\right)^{-1}(A B-1)\left(1+L_{n}\right)^{-1}\left(L_{n} B L_{n}-A^{-1}\right) .
\end{aligned}
$$

This completes the proof.

2.2. Solution formula. To extract scalar solution formulae, some algebraic terminology will be useful.

A one-dimensional operator $T \in \mathcal{L}(E)$ is an operator whose range is contained in a one-dimensional subspace of $E$. Such an operator $T$ is always of the form $a \otimes c$ for a vector $c \in E$ and a functional $a \in E^{\prime}$, where

$$
a \otimes c(x):=\langle x, a\rangle c \quad \forall x \in E .
$$

$\left(\langle\right.$,$\rangle is the usual dual pairing: for the functional a \in E^{\prime},\langle x, a\rangle$ is the evaluation of $a$ at the vector $x \in E$.)

Then the functional $\tau$ is well-defined by

$$
\tau(T)=\langle c, a\rangle \text { for any representation } T=a \otimes c .
$$

Fixing $a \in E^{\prime}$, we define $\mathcal{S}_{a}=\{a \otimes c \mid c \in E\}$, the subalgebra of $\mathcal{L}(E)$ of onedimensional operators whose behaviour is governed by the functional $a$. The restriction 
of $\tau$ to $\mathcal{S}_{a}$ has three crucial properties. It is (1) linear, (2) continuous, and (3) multiplicative; i.e., $\tau\left(T_{1} \circ T_{2}\right)=\tau\left(T_{1}\right) \tau\left(T_{2}\right)$ for $T_{1}, T_{2} \in \mathcal{S}_{a}$.

Starting from the $\mathcal{L}$-solution $V_{n}$ obtained in Theorem 2.1, we now derive $\mathbb{C}$-solutions of (2) according to the following ansatz.

(i) Assume that the solution $L_{n}(x, y)$ of (4) and the operators $A, B$ are coupled in the sense that for some (constant) functional $a \in E^{\prime}$ we have $A L_{n}(x, y) B-L_{n}(x, y) \in \mathcal{S}_{a}$ for all $n \in \mathbb{Z},(x, y) \in \mathbb{R}^{2}$. This means that there is a vector $c_{n}(x, y) \in E$ such that $A L_{n}(x, y) B-L_{n}(x, y)=a \otimes c_{n}(x, y) \forall n \in \mathbb{Z},(x, y) \in \mathbb{R}^{2}$.

(ii) Applying the functional $\tau$ to the $\mathcal{L}$-solution $V_{n}=\left(1+L_{n}\right)^{-1}\left(A L_{n} B-L_{n}\right)=$ $a \otimes\left(\left(1+L_{n}\right)^{-1} c_{n}\right)$, we obtain the $\mathbb{C}$-function $v_{n}:=\tau\left(V_{n}\right)=\left\langle\left(1+L_{n}\right)^{-1} c_{n}, a\right\rangle$. It remains to verify that $v_{n}$ solves (2). This follows from the above mentioned properties of $\left.\tau\right|_{\mathcal{S}_{a}}$.

For a detailed justification of this ansatz we refer to [11].

REMARK 2.2. Note that in (i) it already suffices to assume, say, $A L_{0} B-L_{0}=$ $a \otimes c_{0}(x, y)$. From (4) and the fact that $A$ and $B$ commute, it then follows easily that

$$
A L_{n} B-L_{n}=(A B)^{n}\left(A L_{0} B-L_{0}\right)=(A B)^{n}\left(a \otimes c_{0}(x, y)\right)=a \otimes\left((A B)^{n} c_{0}(x, y)\right),
$$

which implies $A L_{n} B-L_{n}=a \otimes c_{n}(x, y)$ for all $n \in \mathbb{Z}$.

Let us sum up.

THEOREM 2.3. Let $E$ be a Banach space and $A, B \in \mathcal{L}(E)$ with $[A, B]=0$. Moreover, let $C \in \mathcal{L}(E)$ be coupled with $A, B$ such that $A C B-C=a \otimes c$.

Then

$$
\begin{aligned}
v_{n}= & \left\langle\left(1+M_{n} C\right)^{-1} M_{n} c, a\right\rangle \\
& \text { with } M_{n}(x, y)=(A B)^{n} \exp \left(\left(A-B^{-1}\right) x+\left(A^{-1}-B\right) y\right)
\end{aligned}
$$

is a solution of the two-dimensional Toda lattice (2) on

$$
\Omega=\left\{(x, y) \mid 1+M_{n}(x, y) C \text { is invertible } \forall n \in \mathbb{Z}\right\} .
$$

Proof. Observe that $L_{n}=M_{n} C$ satisfies the hypothesis of Theorem 2.1. Thus $V_{n}=\left(1+M_{n} C\right)^{-1}\left(A M_{n} C B-M_{n} C\right)$ is a solution of (3). Since $V_{n} \in \mathcal{S}_{a}$ by the coupling condition $\left.\tau\right|_{\mathcal{S}_{a}}$ is linear, continuous, and multiplicative, $v_{n}=\tau\left(V_{n}\right)$ solves (2). The following calculation finishes the proof.

$$
\begin{aligned}
v_{n}=\tau\left(V_{n}\right) & =\tau\left(\left(1+M_{n} C\right)^{-1}\left(A M_{n} C B-M_{n} C\right)\right) \\
& =\tau\left(\left(1+M_{n} C\right)^{-1} M_{n}(A C B-C)\right)=\tau\left(\left(1+M_{n} C\right)^{-1} M_{n}(a \otimes c)\right) \\
& =\left\langle\left(1+M_{n} C\right)^{-1} M_{n} c, a\right\rangle .
\end{aligned}
$$

REMARK 2.4. Theorem 2.3 can be understood as a linearization procedure since we have reduced the solution of the nonlinear two-dimensional Toda lattice (2) to the solution of the set (4) of linear operator equations in $\mathcal{L}$.

2.3. Improvement of the solution formula. If $\tau$ is induced by a trace, we can improve our solution formula (8) considerably. This will be important for the countable 
superposition of line-solitons in Section 4. Here we start with the finite-dimensional case.

Proposition 2.5. Assume the situation of Theorem 2.3 and $E=\mathbb{C}^{N}$. Then the solution (8) is given by

$$
v_{n}=\frac{\operatorname{det}\left(1+M_{n+1} C\right)}{\operatorname{det}\left(1+M_{n} C\right)}-1 .
$$

Proof. We can identify operators on $E=\mathbb{C}^{N}$ with $N \times N$ matrices. Now we observe that for one-dimensional operators (a) the usual trace $\operatorname{tr}$ coincides with $\tau$ and (b) we have $1+\operatorname{tr}(T)=\operatorname{det}(1+T)$.

Using the notation of Theorem 2.3, we calculate

$$
\begin{aligned}
v_{n}= & \left\langle\left(1+M_{n} C\right)^{-1} M_{n} c, a\right\rangle \\
= & \tau\left(\left(1+M_{n} C\right)^{-1} M_{n}(a \otimes c)\right) \\
= & \operatorname{tr}\left(\left(1+M_{n} C\right)^{-1} M_{n}(a \otimes c)\right) \\
= & \left(1+\operatorname{tr}\left(\left(1+M_{n} C\right)^{-1} M_{n}(a \otimes c)\right)\right)-1 \\
= & \operatorname{det}\left(1+\left(1+M_{n} C\right)^{-1} M_{n}(a \otimes c)\right)-1 \\
= & \operatorname{det}\left(1+\left(1+M_{n} C\right)^{-1}\left(A M_{n} C B-M_{n} C\right)\right)-1 \\
= & \operatorname{det}\left(\left(1+M_{n} C\right)^{-1}\left(1+A M_{n} C B\right)\right)-1 \\
= & \frac{\operatorname{det}\left(1+A M_{n} C B\right)}{\operatorname{det}\left(1+M_{n} C\right)}-1 \\
= & \frac{\operatorname{det}\left(1+B A M_{n} C\right)}{\operatorname{det}\left(1+M_{n} C\right)}-1 \\
= & \frac{\operatorname{det}\left(1+M_{n+1} C\right)}{\operatorname{det}\left(1+M_{n} C\right)}-1 .
\end{aligned}
$$

REMARK 2.6. Later we will need to extend Proposition 2.5 to infinite-dimensional Banach spaces. To this end, the main point is to interpret the expression $\operatorname{det}(1+T)$, where $T$ is an appropriate operator. Roughly speaking, there is a natural choice if $T$ has finite-dimensional range. But in our case, the difficulty is that in line (9) the operators $M_{n} C$ need not be of this kind.

The extension of the notion of determinants is one of the topics of the theory of Banach operator ideals (see [27]). We shall come back to this in Section 4.

\section{Examples.}

3.1. Line-solitons. Consider the finite case, $E=\mathbb{C}^{N}$, and take diagonal matrices $A=\operatorname{diag}\left\{p_{j} \mid j=1, \ldots, N\right\}, \quad B=\operatorname{diag}\left\{1 / q_{j} \mid j=1, \ldots, N\right\} \quad$ with $0<p_{N}<\ldots<p_{1}<$ $q_{1}<\ldots<q_{N}$. In this case it is easy to calculate explicitly a matrix $C$ which satisfies $A C B-C=a \otimes c$, namely

$$
C=\left(\frac{a_{j} c_{i}}{p_{i} / q_{j}-1}\right)_{i, j=1}^{N}
$$


Then Proposition 2.5 implies that

$$
v_{n}=\frac{\operatorname{det}\left(1+M_{n+1} C\right)}{\operatorname{det}\left(1+M_{n} C\right)}-1
$$

solves (2). The resulting solution describes the superposition of $N$ line-solitons; it depends on the $3 N$ parameters $p_{j}, q_{j}, a_{j} \cdot c_{j}(j=1, \ldots, N)$.

The line-solitons (11) are always regular. The reader finds a proof in Appendix A and a comparison to the representation by Casorati determinants (as used in [5], [6]) in Appendix B. Some recent results on the interaction patterns of line-solitons in the related case of the KP equation can be found in [14], [15].

3.2. Resonant interactions. The appearance of resonance phenomena for the two-dimensional Toda lattice is not surprising since its continuum limit yields the Kadomtsev-Petviashvili equation (see [25]) for which resonance phenomena are well known and intensively studied (see, for example, [16], [17], [18], and [19]).

In our framework, resonantly interacting solitons arise from inserting diagonal matrices $A=\operatorname{diag}\left\{p_{j} \mid j=1, \ldots, N\right\}$ and $B=\operatorname{diag}\left\{1 / q_{j} \mid j=1, \ldots, N\right\}$ in the limiting case in which some of the entries coincide $\left(0<p_{N} \leq \ldots \leq p_{1}<q_{1} \leq \ldots \leq q_{N}\right)$.

In the sequel we shall take a closer look at the smallest case of interest, $N=2$. Assume that

$$
A=\left(\begin{array}{cc}
p_{1} & 0 \\
0 & p_{2}
\end{array}\right) \quad \text { and } \quad B=\left(\begin{array}{cc}
1 / q_{1} & 0 \\
0 & 1 / q_{2}
\end{array}\right) \text { with } p_{1}=p_{2}=: p .
$$

Then it turns out that the matrix $C$ with $A C B-C=a \otimes c$, that we calculated in (10), itself is one-dimensional, namely $C=\hat{a} \otimes c$ with $\hat{a}_{j}=a_{j} /\left(p / q_{j}-1\right)$. Thus

$$
\operatorname{det}\left(1+M_{n} C\right)=\operatorname{det}\left(1+\hat{a} \otimes M_{n} c\right)=1+\left\langle M_{n} c, \hat{a}\right\rangle=1+l_{n} \sum_{j=1,2} m_{n}^{(j)}
$$

where we have set $\hat{a}_{j} c_{j}=\exp \left(-\beta_{j}\right)$ and

$$
l_{n}(x, y)=p^{n} \exp (p x+y / p), \quad m_{n}^{(j)}(x, y)=\left(q_{j}^{n} \exp \left(q_{j} x+y / q_{j}+\beta_{j}\right)\right)^{-1} .
$$

To give a rough idea of the asymptotic analysis, we observe that, for any fixed value of $y$, we may expect a soliton contribution to the solution in the following regions: 1. $l_{n} m_{n}^{(1)} \approx c$ and $l_{n} m_{n}^{(2)} \approx 0,2 . l_{n} m_{n}^{(1)} \approx 0$ and $l_{n} m_{n}^{(2)} \approx c$, and 3. $m_{n}^{(1)} \approx m_{n}^{(2)}$ and $l_{n} \approx \infty$.

The computer graphic confirms these heuristics, which can be treated rigorously without much effort. More careful analysis shows that also for arbitrary $N$ the choice $A=p I, B$ a diagonal matrix with $N$ different entries leads to tree-like structures with $N+1$ solitons, one of the branches pointing to $-\infty$, all the others to $+\infty$ in the $x$-direction. Vice versa, if $A$ is a diagonal matrix with $N$ different entries, $B=(1 / q) I$ the picture is reflected at the axis $x=0$.

4. Countable superpositions of line-solitons. As our main application, we give an answer to the question whether it is possible to superpose countably many line-solitons. This question has been raised by Gesztesy et al. [20]. Results for the one-dimensional 

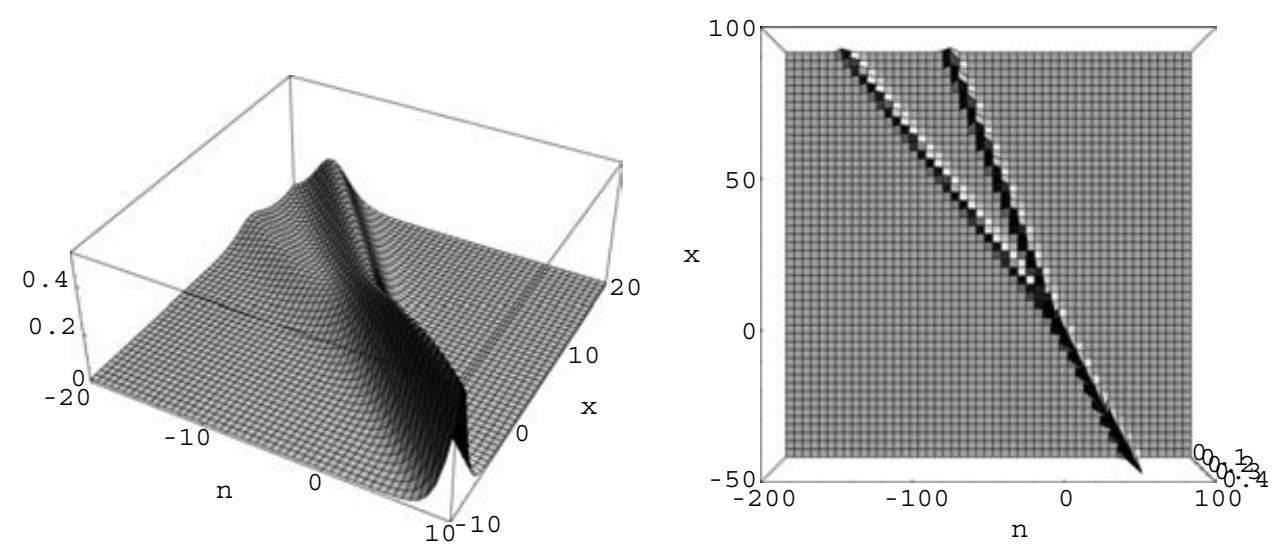

Figure 1. The function $f_{n}(x, y)=\left(1+v_{n+1}(x, y)\right) /\left(1+v_{n}(x, y)\right)-1$ ('the force') is plotted for $y=0$ from two different points of view. For simplicity we have interpreted $n$ as a continuous variable. The parameters are: $p=0.5, q_{1}=1.2$ and $q_{2}=2$.

Toda lattice are due to Gesztesy and Renger [21]. To the best of our knowledge, Theorem 4.3 is the first result of this kind for the two-dimensional Toda lattice.

More precisely, the problem is the following: given sequences

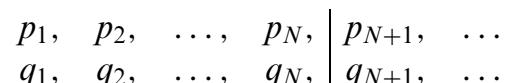

the first $N$ parameters always define an $N$-line-soliton configuration $v_{n}^{(N)}$ as in Section 3.1. Now one would like to find conditions on these sequences that guarantee the existence of the limit $v=\lim _{N \rightarrow \infty} v_{n}^{(N)}$, and, even more, that this limit $v$ still solves (2).

Within the framework of Theorem 2.3, the answer becomes rather transparent.

Let $E=\ell_{2}$ and consider the diagonal operators $A\left(\xi_{j}\right)_{j}=\left(p_{j} \xi_{j}\right)_{j}, B\left(\xi_{j}\right)_{j}=\left(\xi_{j} / q_{j}\right)_{j}$ given by the bounded sequence $\left(p_{j}\right)_{j}$ and the sequence $\left(q_{j}\right)_{j}$ with $\inf _{j}\left|q_{j}\right|>0$. Obviously $A, B \in \mathcal{L}\left(\ell_{2}\right)$. For $a=\left(a_{j}\right)_{j}, c=\left(c_{j}\right)_{j} \in \ell_{2}$ we define

$$
C\left(\xi_{j}\right)_{j}=\left(\sum_{j} \frac{a_{j} c_{i}}{p_{i} / q_{j}-1} \xi_{j}\right)_{i}
$$

which also defines a bounded operator if we assume $\alpha:=\inf _{i, j}\left|p_{i} / q_{j}-1\right|>0$. Indeed, by Hölder's inequality we have

$$
\begin{aligned}
\left\|C\left(\xi_{j}\right)_{j}\right\|_{2}^{2} & =\sum_{i}\left|\sum_{j} \frac{a_{j} c_{i}}{p_{i} / q_{j}-1} \xi_{j}\right|^{2} \leq \sum_{i}\left(\sum_{j}\left|\frac{a_{j} c_{i}}{p_{i} / q_{j}-1} \xi_{j}\right|\right)^{2} \\
& \leq \frac{1}{\alpha^{2}} \sum_{i}\left(\sum_{j}\left|c_{i}\right|\left|a_{j} \xi_{j}\right|\right)^{2}=\frac{1}{\alpha^{2}} \sum_{i}\left|c_{i}\right|^{2}\left(\sum_{j}\left|a_{j} \xi_{j}\right|\right)^{2} \\
& =\frac{1}{\alpha^{2}}\left\|\left(c_{i}\right)_{i}\right\|_{2}^{2}\left\|\left(a_{j} \xi_{j}\right)_{j}\right\|_{1}^{2} \leq \frac{\left\|\left(c_{i}\right)_{i}\right\|_{2}^{2}\left\|\left(a_{j}\right)_{j}\right\|_{2}^{2}}{\alpha^{2}}\left\|\left(\xi_{j}\right)_{j}\right\|_{2}^{2} ;
\end{aligned}
$$


in other words $\|C\| \leq\|a\|\|c\| / \alpha$. Finally, it can be easily checked that $A C B-C=$ $a \otimes c$. Now we can apply Theorem 2.3. Note that

$$
M_{n}(x, y)\left(\xi_{j}\right)_{j}=\left(\left(p_{j} / q_{j}\right)^{n} \exp \left(\left(p_{j}-q_{j}\right) x+\left(1 / p_{j}-1 / q_{j}\right) y\right) \xi_{j}\right)_{j}
$$

As a result we have proved the following result.

THEOREM 4.1. Let $\left(p_{j}\right)_{j}$ be a bounded sequence and $\left(q_{j}\right)_{j}$ a sequence bounded away from zero. Assume $\inf _{i, j}\left|p_{i} / q_{j}-1\right|>0$. Choose a, $c \in \ell_{2}$ arbitrarily and define $C$ as in (12). Then

$$
v_{n}=\left\langle\left(1+M_{n} C\right)^{-1} M_{n} c, a\right\rangle
$$

is a solution of the two-dimensional Toda lattice (2) describing the countable superposition of line-solitons corresponding to the parameters $p_{j}, q_{j}, a_{j} \cdot c_{j}$.

We can even obtain a similar simplification of our solution formula as in Section 2.3. To this end, we have to generalize our arguments to infinite-dimensional Banach spaces; compare Remark 2.6.

LemMA 4.2. Under the assumptions of Theorem 4.1, the operator $C$ defined in (12) is trace class.

Proof. By $\mathcal{B}_{1}\left(\ell_{2}\right)$ we denote the trace class operators on $\ell_{2}$. Consider the elementary operator $\Phi \in \mathcal{L}\left(\mathcal{B}_{1}\left(\ell_{2}\right)\right)$ which is defined by

$$
\Phi(X):=A X B-X \text { for } X \in \mathcal{B}_{1}\left(\ell_{2}\right) .
$$

Then $\operatorname{spec}(\Phi)=\operatorname{spec}(A) \cdot \operatorname{spec}(B)-1$ (see for example [26]). Thus the assumption $\inf _{i, j}\left|p_{i} / q_{j}-1\right|>0$ yields $0 \notin \operatorname{spec}(\Phi)$, and $\Phi$ is invertible in $\mathcal{L}\left(\mathcal{B}_{1}\left(\ell_{2}\right)\right)$. Since $a \otimes c$ surely is trace class (it is even one-dimensional), also $D:=\Phi^{-1}(a \otimes c)$ is trace class. Now $D$ solves $A D B-D=a \otimes c$. Calculating its matrix entries with respect to the standard basis, we derive $D=C$. See [12, Section 4] for more details.

It can be shown (see [27]) that $T$ is trace class if and only if there is a representation $T=\sum_{j=1}^{\infty} a_{j} \otimes c_{j}$ with $\sum_{j=1}^{\infty}\left\|a_{j}\right\| \cdot\left\|c_{j}\right\|<\infty$; i.e., if $T$ is an infinite sum of onedimensional operators.

Then the Fredholm determinant is given by

$$
\operatorname{det}_{1}(1+T)=1+\sum_{n=1}^{\infty} \frac{1}{n !} \sum_{i_{1}=1}^{\infty} \ldots \sum_{i_{n}=1}^{\infty} \operatorname{det}\left(\begin{array}{ccc}
\left\langle c_{i_{1}}, a_{i_{1}}\right\rangle & \ldots & \left\langle c_{i_{1}}, a_{i_{n}}\right\rangle \\
\vdots & & \vdots \\
\left\langle c_{i_{n}}, a_{i_{1}}\right\rangle & \ldots & \left\langle c_{i_{n}}, a_{i_{n}}\right\rangle
\end{array}\right)
$$

and the corresponding trace by

$$
\operatorname{tr}_{1}(T)=\sum_{j=1}^{\infty}\left\langle c_{j}, a_{j}\right\rangle,
$$

where $T=\sum_{j=1}^{\infty} a_{j} \otimes c_{j}$ is an arbitrary representation of $T$.

Since $\operatorname{tr}_{1}$ coincides with $\tau$ on $\mathcal{S}_{a}$, the arguments of Section 2.3 carry over and yield the following result. 
THEOREM 4.3. The following reformulation of the solution (13) in terms of the Fredholm determinant det $_{1}$ holds:

$$
v_{n}=\frac{\operatorname{det}_{1}\left(1+M_{n} C\right)}{\operatorname{det}_{1}\left(1+M_{n+1} C\right)}-1 .
$$

REMARK 4.4. The conditions can even be sharpened. In the sequel we only assume that $\left(p_{j}\right)_{j},\left(1 / q_{j}\right)_{j}$ are bounded sequences with $p_{j}, 1 / q_{j}>1$. Since the quality of the operator $C$ was decisive in deriving Theorem 4.3 , the main idea is to find conditions on the sequences $a, c$ such that $C$ still is an operator admitting an appropriate determinant.

Let us mention two results.

1. If $\left(a_{j} / \sqrt{1 / q_{j}^{2}-1}\right)_{j},\left(c_{j} / \sqrt{p_{j}^{2}-1}\right)_{j} \in \ell_{2}$, then a sophisticated argument shows that $C$ factors through $\ell_{1}, L_{2}[0, \infty)$ and $\ell_{\infty}$. Using Grothendieck's theorem and a deep result of White [28] on operators with summable eigenvalues, we can show that Theorem 4.3 still holds, but with the spectral determinant $\operatorname{det}_{\lambda}$.

2. If $\left(a_{j}\right)_{j} \in \ell_{\infty},\left(c_{j} /\left(p_{j}^{2}-1\right)\right)_{j} \in \ell_{1}$, then it turns out that $C \in \mathcal{N}\left(\ell_{1}\right)$. Therefore, switching over to operators on the Banach space $\ell_{1}$, again Theorem 4.3 holds, where we now use the nuclear determinant $\operatorname{det}_{\mathcal{N}}$ on the nuclear operators $\mathcal{N}\left(\ell_{1}\right)$.

Statements 1 . and 2. can be proved by similar arguments as in [12, Theorem 6.4] and Proposition 6.6.

Note that nuclear operators on $\ell_{1}$ are defined analogously to the characterization of trace class operators as infinite sums of one-dimensional operators. But observe that the corresponding nuclear $\operatorname{trace} \operatorname{tr}_{\mathcal{N}}$ behaves rather strangely. Namely, a nilpotent operator $S \in \mathcal{N}\left(\ell_{1}\right)$ can be constructed with $\operatorname{tr}_{\mathcal{N}}(S)=1$ (see [29]).

Already this example shows that traces and determinants are not straightforward objects in general. We refer to [12] for an introduction to this topic, and to [27], [30] for further reading.

\section{Appendix}

\section{A. Regularity of line-solitons.}

LEMmA A.1. For $a_{j} c_{j}>0$, the line-soliton (11) is regular.

Proof. Recall that for the line-soliton (11), we have $0<p_{N}<\ldots<p_{1}<q_{1}<\ldots<$ $q_{N}$ and

$$
M_{n} C=\left(\frac{a_{j} c_{i}}{p_{i} / q_{j}-1}\left(p_{j} / q_{j}\right)^{n} \exp \left(\left(p_{j}-q_{j}\right) x+\left(1 / p_{j}-1 / q_{j}\right) y\right)\right)_{i, j=1}^{N}
$$

Therefore a well-known expansion rule yields

$$
\operatorname{det}\left(1+M_{n} C\right)=1+\sum_{k=1}^{N} \sum_{\substack{j_{1}, \ldots, j_{k}=1 \\ j_{1}<\ldots<j k}}^{N} \operatorname{det}\left(\left(M_{n} C\right)^{j_{1}, \ldots, j_{k}}\right)
$$


where $\left(M_{n} C\right)^{j_{1}, \ldots, j_{k}}$ denotes the matrix obtained from $M_{n} C$ by deleting the $j_{1}, \ldots, j_{k}$-th rows and columns. Now with $f_{j}(n, x, y):=\left(p_{j} / q_{j}\right)^{n} \exp \left(\left(p_{j}-q_{j}\right) x+\left(1 / p_{j}-1 / q_{j}\right) y\right)$,

$$
\begin{aligned}
\operatorname{det}\left(M_{n} C\right) & =\prod_{j=1}^{N}\left(a_{j} c_{j} f_{j}(n, x, y)\right) \cdot \operatorname{det}\left(\frac{1}{p_{i} / q_{j}-1}\right)_{i, j=1}^{N} \\
& =\prod_{j=1}^{N} \frac{a_{j} c_{j}}{p_{j} / q_{j}-1} f_{j}(n, x, y) \prod_{\substack{i, j=1 \\
i<j}}^{N} \frac{\left(p_{i}-p_{j}\right)\left(1 / q_{i}-1 / q_{j}\right)}{\left(p_{i} / q_{j}-1\right)\left(p_{j} / q_{i}-1\right)},
\end{aligned}
$$

where we refer to [31] for the calculation of the latter determinant. From $a_{j} c_{j}>0$, we conclude $\operatorname{det}\left(M_{n} C\right) \geq 0$, and of course the same is valid for the determinant of any minor $\left(M_{n} C\right)^{j_{1}, \ldots, j_{k}}$. Thus $\operatorname{det}\left(1+M_{n} C\right) \geq 1$, which shows that the line-soliton (11) is regular.

B. Connection to Casorati determinants. In the literature, line-solitons are derived in terms of Casorati determinants

$$
v_{n}=\frac{\operatorname{det}\left(\left(\varphi_{i}(n+j)\right)_{i, j=1}^{N}\right)}{\operatorname{det}\left(\left(\varphi_{i}(n+j-1)\right)_{i, j=1}^{N}\right)}-1
$$

with $\varphi_{i}(n)=\left(p_{i}\right)^{n} \exp \left(p_{i} x+y / p_{i}+\alpha_{i}\right)+\left(q_{i}\right)^{n} \exp \left(q_{i} x+y / q_{i}+\beta_{i}\right)$. More precisely, the representation (15) follows from [5] combined with [6] if one takes into account the straightforward transformations linking the different versions of the equations under consideration. In the following lemma we show how to translate (15) into our framework.

LEMMA B.1. Every $v_{n}$ as in (15) can be realized as one of the solutions constructed in Theorem 2.3.

Proof. We explicitly construct a line-soliton (11) leading to the same solution as (15). Choose $A=\operatorname{diag}\left\{p_{j} \mid j=1, \ldots, N\right\}, B=\operatorname{diag}\left\{1 / q_{j} \mid j=1, \ldots, N\right\}$. In addition, we consider the Vandermonde matrices $V=\left(p_{i}^{j-1}\right)_{i, j=1}^{N}$ and $W=\left(q_{i}^{j-1}\right)_{i, j=1}^{N}$. For the following decisive but rather technical fact we refer to the forthcoming work [32]. The expression $A\left(V W^{-1}\right) B-\left(V W^{-1}\right)$ is one-dimensional.

Set $C=A_{0} B_{0}^{-1} \cdot\left(V W^{-1}\right)$ with $A_{0}=\operatorname{diag}\left\{\exp \left(\alpha_{j}\right) \mid j=1, \ldots, N\right\}$ and $B_{0}=$ $\operatorname{diag}\left\{\exp \left(\beta_{j}\right) \mid j=1, \ldots, N\right\}$. Then the expression $A C B-C$ is still one-dimensional: i.e., there exist vectors $a, c$ such that $A C B-C=a \otimes c$. By Theorem 2.3, we obtain the following solution $v_{n}$ of (2),

$$
v_{n}=\left\langle\left(1+M_{n} C\right)^{-1} M_{n} c, a\right\rangle
$$

with $M_{n}=(A B)^{n} \exp \left(\left(A-B^{-1}\right) x+\left(A^{-1}-B\right) y\right)$. Since we are in the finite-dimensional case, we may repeat the arguments of Section 3.1 to see that

$$
v_{n}=\frac{\operatorname{det}\left(1+M_{n+1} C\right)}{\operatorname{det}\left(1+M_{n} C\right)}-1 .
$$


Using $\quad M_{n} C=G_{n}^{-1} F_{n} \cdot V W^{-1} \quad$ where $\quad G_{n}=B^{-n} \exp \left(B^{-1} x+B y\right) B_{0}$ and $F_{n}=$ $A^{n} \exp \left(A x+A^{-1} y\right) A_{0}$, we infer that

$$
\operatorname{det}\left(1+M_{n} C\right)=\operatorname{det}\left(G_{n}^{-1}\left(F_{n} V+G_{n} W\right) W^{-1}\right)=\frac{\operatorname{det}\left(F_{n} V+G_{n} W\right)}{\operatorname{det}\left(G_{n} W\right)} .
$$

We easily check that $\operatorname{det}\left(F_{n} V+G_{n} W\right)=\operatorname{det}\left(\left(\varphi_{i}(n+j-1)\right)_{i, j=1}^{N}\right)$. Thus

$$
1+v_{n}=\frac{1}{\operatorname{det}(B)} \quad \frac{\operatorname{det}\left(\left(\varphi_{i}(n+j)\right)_{i, j=1}^{N}\right)}{\operatorname{det}\left(\left(\varphi_{i}(n+j-1)\right)_{i, j=1}^{N}\right)} .
$$

Since (2) admits a multiplicative transformation group (as an equation for $1+v_{n}$ ), we may eliminate $1 / \operatorname{det}(B)$, which means that $v_{n}$ generates the same solution as (15).

\section{REFERENCES}

1. C. Schiebold, An operator theoretic approach to the Toda lattice equation, Physica $D$ 122 (1998), 37-61. $180-192$.

2. C. Schiebold, On negatons of the Toda lattice, J. Nonlin. Math. Phys. 10, Suppl. 2 (2003),

3. G. Darboux, Leçons sur la théorie des surfaces (Gauthiers-Villars, Paris, 1915).

4. M. J. Ablowitz and J. Villarroel, Solutions to the $2+1$ Toda equation, J. Phys. A 27 (1994), 931-941.

5. R. Hirota, M. Ito and F. Kako, Two-dimensional Toda lattice equations, Progr. Theor. Phys. Suppl. 94 (1988), 42-58.

6. R. Hirota, Y. Ohta and J. Satsuma, Wronski structure of solutions for soliton equations, Progr. Theor. Phys. Suppl. 94 (1988), 59-72. 1991).

7. V. B. Matveev and M. A. Salle, Darboux transformations and solitons (Springer-Verlag,

8. M. Mañas, L. Martínez Alonso and E. Medina, Dressing methods for geometric nets: I. Conjugate nets, J. Phys. A. 33 (2000), 2871-2894.

9. M. Mañas, L. Martínez Alonso and E. Medina, Dressing methods for geometric nets: II. Orthogonal and Egorov nets, J. Phys. A. 33 (2000), 7181-7206.

10. J. J. C. Nimmo and R. Willox. Darboux transformations for the two-dimensional Toda system. Proc. Roy. Soc. London Ser. A 453 (1997), 2497-2525.

11. H. Aden and B. Carl, On realizations of solutions of the $\mathrm{KdV}$ equation by determinants on operator ideals, J. Math. Phys. 37 (1996), 1833-1857.

12. B. Carl and C. Schiebold, Nonlinear equations in soliton physics and operator ideals, Nonlinearity 12 (1999), 333-364.

13. V. A. Marchenko, Nonlinear equations and operator algebras (Reidel, Dordrecht, 1988).

14. P. Peterson and E. van Groesen, A direct and inverse problem for wave crests modelled by interactions of two solitons, Physica D 141 (2000), 316-332.

15. P. Peterson, Reconstruction of multi-soliton interactions using crest data for $(2+1)$ dimensional KdV type equations, Physica D 171 (2002), 221-235.

16. G. Biondini and Y. Kodama, On a family of solutions of the Kadomtsev-Petviashvili equation which also satisfy the Toda lattice hierarchy, J. Phys. A. 36 (2003), 10519-10536.

17. E. Medina, An $N$ soliton resonance solution for the KP equation: Interaction with change of form and velocity, Lett. Math. Phys. 62 (2002), 91-99.

18. J. Miles, Resonantly interacting solitary waves, J. Fluid Mech. 79 (1976), 171-179.

19. K. Ohkuma and M. Wadati, The Kadomtsev-Petviashvili equation: the trace method and the soliton resonances, J. Phys. Soc. Japan 52 (1982), 749-760.

20. F. Gesztesy, W. Karwowski and Z. Zhao, Limits of soliton solutions, Duke Math. J. 68 (1992), 101-150.

21. F. Gesztesy and W. Renger, New classes of Toda soliton solutions, Commun. Math. Phys. 184 (1997), 27-50.

22. A. Pietsch, Operator ideals (VEB Deutscher Verlag der Wissenschaften, 1978). 
23. B. Carl and I. Stephani, Entropy, compactness and the approximation of operators (Cambridge Tracts in Mathematics, No. 98, Cambridge University Press, 1990).

24. B. Carl and C. Schiebold, Ein direkter Ansatz zur Untersuchung von Solitonengleichungen. Jber. d. Dt. Math.-Verein. 102 (2000), 102-148.

25. A. V. Mikhailov, Integrability of a two-dimensional generalization of the Toda chain, Sov. Phys. JETP Lett. 30 (1979), 414-418.

26. A. Eschmeier, Tensor products and elementary operators, J. Reine Angew. Math. 390 (1988), 47-66.

27. A. Pietsch. Eigenvalues and s-numbers (Cambridge University Press, 1987).

28. M. C. White, Analytic multivalued functions and spectral trace, Math. Ann. 304 (1996), 669-683.

29. P. Enflo. A counterexample to the approximation problem in Banach spaces, Acta Math. 130 (1973), 309-317.

30. B. Simon, Trace ideals and their applications (Cambridge University Press, 1979).

31. G. Pólya and G. Szegö, Aufgaben und Lehrsätze zur Analysis II (Springer-Verlag, 1976).

32. C. Schiebold, Habilitationsschrift (Jena, 2004). 
